\title{
Melatonin does not attenuate dynamic cardiovascular and cerebrovascular reflex responses to acute hypotension in healthy men
}

\author{
Jiyoun Bang, Yong Seok Park, Sung-Moon Jeong, Jun-Gol Song, Young-Kug Kim, and Gyu-Sam Hwang
}

Department of Anesthesiology and Pain Medicine, Asan Medical Center, University of Ulsan College of Medicine, Seoul, Korea

Background: Melatonin has been shown to attenuate the reflex sympathetic increases that arise in response to orthostatic challenges. We tested the hypothesis that the attenuated sympathetic increase induced by melatonin premedication may weaken the arterial blood pressure (ABP) preserving the capability during acute hypotension, thereby altering dynamic cerebral autoregulation and causing a further decrease in cerebral blood flow (CBF).

Methods: Acute hypotension was induced in 12 healthy subjects by releasing bilateral thigh cuffs before and after an oral dose of melatonin $(0.2 \mathrm{mg} / \mathrm{kg})$. Heart rate (HR), arterial blood pressure (ABP), Modelflow estimate of cardiac output (CO), total peripheral resistance (TPR) and cerebral blood flow velocity (CBFV) by transcranial Doppler were measured.

Results: Steady state HR, the mean arterial pressure and CBFV were not altered 60 minutes after melatonin ingestion. Reduced systolic arterial pressure ( $\triangle \mathrm{SAP})$, changes in HR $(\Delta \mathrm{HR}), \mathrm{CO}(\Delta \mathrm{CO})$, and TPR $(\Delta \mathrm{TPR}), \Delta \mathrm{HR} / \Delta \mathrm{SAP}$ and percentage restoration of SAP were not affected after a temporal decrease in ABP induced by thigh cuff release. In the cerebral circulation, melatonin did not affect changes in CBFV, cerebrovascular resistance index, the rate of regulation and percentage restoration of CBFV following a sudden decrease in $\mathrm{ABP}$.

Conclusions: Contrary to our hypothesis, melatonin did not affect the rapid vasodilatory and recovery responses of cardiovascular and dynamic cerebral autoregulation. These results suggest that melatonin premedication may not impair ABP and CBF preserving capability induced by sudden postural changes or hemorrhage. (Korean J Anesthesiol 2012; 63: 245-252)

Key Words: Blood pressure, Cerebrovascular circulation, Melatonin.

Received: May 4, 2012. Revised: 1st, July 9, 2012; 2nd, July 28, 2012. Accepted: July 28, 2012.

Corresponding author: Gyu-Sam Hwang, M.D., Department of Anesthesiology and Pain Medicine, Laboratory for Cardio Vascular Dynamics, Asan Medical Center, UUCM, 388-1, Pungnap 2-dong, Songpa-gu, Seoul 138-736, Korea. Tel: 82-2-3010-3989, Fax: 82-2-3010-6790, E-mail: kshwang@amc.seoul.kr

(c) This is an open-access article distributed under the terms of the Creative Commons Attribution Non-Commercial License (http:// creativecommons.org/licenses/by-nc/3.0/), which permits unrestricted non-commercial use, distribution, and reproduction in any medium, provided the original work is properly cited. 


\section{Introduction}

Melatonin secreted by the pineal gland has been shown to have analgesic, anxiolytic, and sedative effects [1]. Therefore, these beneficial effects have suggested that melatonin can be utilized as a premedicant [2]. However, the premedication dose of melatonin in clinical trials has varied from $0.05 \mathrm{mg} / \mathrm{kg}$ to $0.2 \mathrm{mg} / \mathrm{kg}$, and is relatively higher than $1-5 \mathrm{mg}$ that general populations usually use [2].

Melatonin has been demonstrated to decrease arterial blood pressure $(\mathrm{ABP})$ in normal and hypertensive rats [3,4]. In humans, $1 \mathrm{mg}$ melatonin decreased ABP and plasma norepinephrine concentration after standing $[5,6]$, and $3 \mathrm{mg}$ attenuated reflex sympathetic increases that respond to orthostatic stress $[7,8]$. Therefore, relatively higher doses of melatonin may make patients more susceptible to acute hypotension. For anesthesiologists, this information is important because anesthetized patients frequently experience hypotension resulting from a rapid change in posture or hemorrhage before and during general anesthesia. Nevertheless, melatonin premedication has been used clinically without validating this possible occurrence.

In addition to static changes in cerebral blood flow (CBF), $\mathrm{CBF}$ also changes rapidly in response to temporary changes in ABP [9]. Dynamic cerebral autoregulation (dCA) refers to this capability of the cerebral vessels to buffer alterations in $\mathrm{CBF}$ induced by "temporary" changes in ABP [10]. Little is known, however, about the effects of melatonin on dCA in humans [11].

We have, therefore, evaluated the effects of melatonin premedication on cardiovascular reflex responses and dCA during acute hypotension. To accomplish this, we used the thigh cuff method to induce sudden hypotension with cerebral hypoperfusion. We hypothesized that melatonin premedication would attenuate the sympathetic increase, weakening $\mathrm{ABP}$ preservation, and thereby, altering dCA that may cause a further decrease in CBF.

\section{Materials and Methods}

\section{Subjects}

Twelve healthy men, with the mean age of $26 \pm 3$ years, volunteered for this study, which was approved by the Institutional Review Board of our hospital. This study is registered with http://cris.cdc.go.kr (No.KCT0000213). All subjects were free of any known cardiovascular or cerebrovascular diseases and were not taking any medications. The experimental protocols were explained verbally to each subject, and written informed consent was obtained from each participant. Prior to the actual experimental day, each subject was familiarized with the equipment and the study protocol and was also evaluated to ensure an adequate transcranial Doppler insonation window for the middle cerebral artery (MCA). The subjects were asked to avoid caffeinated or alcoholic beverages for $12 \mathrm{~h}$ and exhausting physical activity for at least $24 \mathrm{~h}$ prior to testing.

\section{Measurements}

Each subject was connected to an electrocardiograph (Hewlett-Packard 78352A, NV, USA) to measure heart rate (HR) and to a photoplethysmographic cuff on the middle finger of the right hand to obtain noninvasive beat-to-beat ABP (Finometer, Finapres Medical Systems BV, Amsterdam, Netherland). Stroke volume (SV) and cardiac output (CO) were calculated from the ABP waveform using the Modelflow algorithm [12], which incorporates age, sex, height and weight information to estimate aortic cross-sectional area (BeatScope1.1a software; TNOTPD Biomedical Instrumentation). Total peripheral resistance (TPR) was measured as mean arterial pressure (MAP) divided by CO. Modelflow estimates of SV were based on a threeelement model of arterial input impedance [12], which allows for precise estimation of beat-to-beat SV during postural stress and exercise [13,14]. However, since the Modelflow measure of CO has not been calibrated [15], we expressed the changes in $\mathrm{CO}, \mathrm{SV}$ and TPR in either absolute values or as relative changes. A pulse oximeter was applied and capnography was measured via a nasal cannula using a mass spectrometer (Marquette Electronics, WI, USA). A bispectral index monitor (BIS XP ${ }^{\circledR}$; Aspect Medical Systems, Inc., Norwood, MA, USA) was utilized to monitor consciousness.

The middle cerebral artery was insonated by placing a 2-MHz Doppler probe (Companion III, Viasys Healthcare, Madison, WI) over the temporal window. CBF velocity (CBFV) was measured with a probe fixation device to stabilize the Doppler probe during the study period. The envelope of the velocity waveform was recorded at a depth of 45-60 $\mathrm{mm}$. All physiological signals were digitized at $500 \mathrm{~Hz}$ using a commercially available analog-to-digital converter (DI-720U, Dataq Instruments, OH, USA) and were stored for later off-line analysis.

\section{Experimental protocols}

Experimental protocols are summarized in Fig. 1. A hypotensive stimulus was induced by sudden deflation of bilateral thigh cuffs [10]. After measuring baseline control data of ABP and CBFV waveforms at baseline for $6 \mathrm{~min}$, the thigh cuffs were rapidly inflated to $30 \mathrm{mmHg}$ above each subject's systolic arterial pressure (SAP) using a cuff inflator (E20 Rapid Cuff Inflator; Hokanson, Inc., Bellevue, WA), which can inflate and deflate a large cuff less than $0.3 \mathrm{~s}$, respectively. Two minute after 


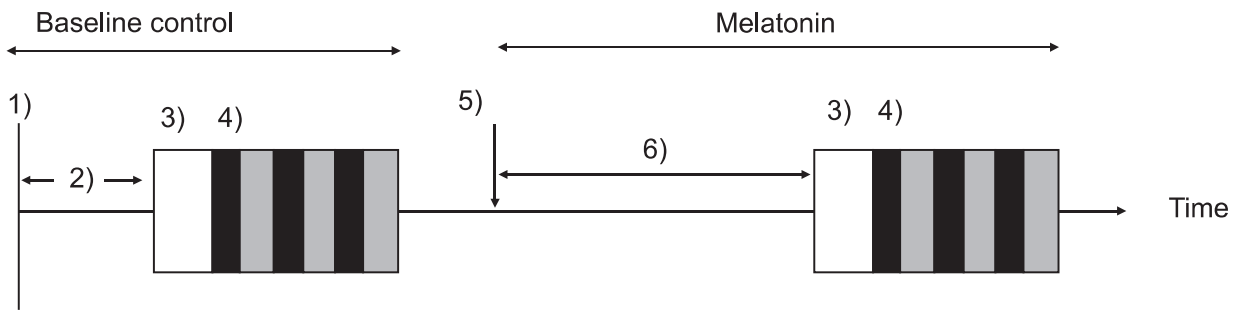

Fig. 1. Summary of experimental protocol. 1) Application of monitor, 2) Supine rest (15 min), 3) $\square$ Data of 6 min resting state, 4) Thigh cuff method, $\square$ thigh cuff inflation $2 \mathrm{~min}$, $\square$ thigh cuff deflation 5 min, 5) Melatonin administration, 6) Supine rest $(60 \mathrm{~min})$. thigh cuffs inflation, the cuffs were rapidly deflated to generate at least a $10 \mathrm{mmHg}$ reduction in $\mathrm{ABP}$. Two or more repeated thigh cuff occlusions were performed at 3 to 5 min intervals, and the mean value in each parameter was determined. If ABP was not reduced more than $10 \mathrm{mmHg}$, the data were excluded from calculations of mean thigh cuff data [16].

Following the baseline control study, each subject was administered $0.2 \mathrm{mg} / \mathrm{kg}$ oral melatonin. This dose was chosen to yield a same concentration of melatonin when it was used as a premedicant in the previous study [17]. Sixty minutes after administration of melatonin, the protocol used for inflation and deflation of the thigh cuffs was repeated and the mean value in each parameter was determined; this 60 min interval was chosen because near peak plasma melatonin concentrations in young, healthy subjects were observed approximately $1 \mathrm{~h}$ after melatonin ingestion [8].

\section{Data analysis}

Beat-to-beat R-R intervals were determined from the $\mathrm{R}$ wave of the electrocardiogram. Systolic, diastolic, and mean blood pressure and mean CBFV were determined from the associated and integrated signals within each cardiac cycle using MATLAB (MathWorks, Natick, MA, USA) and DADiSP software (DSP Development, Cambridge, MA, USA).

\section{Cardiovascular responses to thigh cuff release}

Baseline values of SAP, HR, SV, CO and TPR were obtained by calculating their averages during the $10 \mathrm{~s}$ before thigh cuff release. Reduction in SAP $(\triangle \mathrm{SAP})$ was estimated by subtracting the minimum SAP after thigh cuff release from baseline SAP, and change in $\mathrm{HR}(\triangle \mathrm{HR})$ was estimated by subtracting baseline HR from the maximum HR after thigh cuff release, and these values were used to calculate the increased HR in response to reduced SAP $(\Delta \mathrm{HR} / \Delta \mathrm{SAP})$, an index of arterial cardiac reflex $[18,19]$. The percentage restoration of SAP was shown as restoration/reduction $\times 100$ and calculated as [(recovery SAP - minimum SAP)/(baseline SAP - minimum SAP)] $\times 100(\%)$, where recovery SAP is the average SAP measured in the $10 \mathrm{~s}$ interval between 10 and $20 \mathrm{~s}$ following thigh cuff release [18].

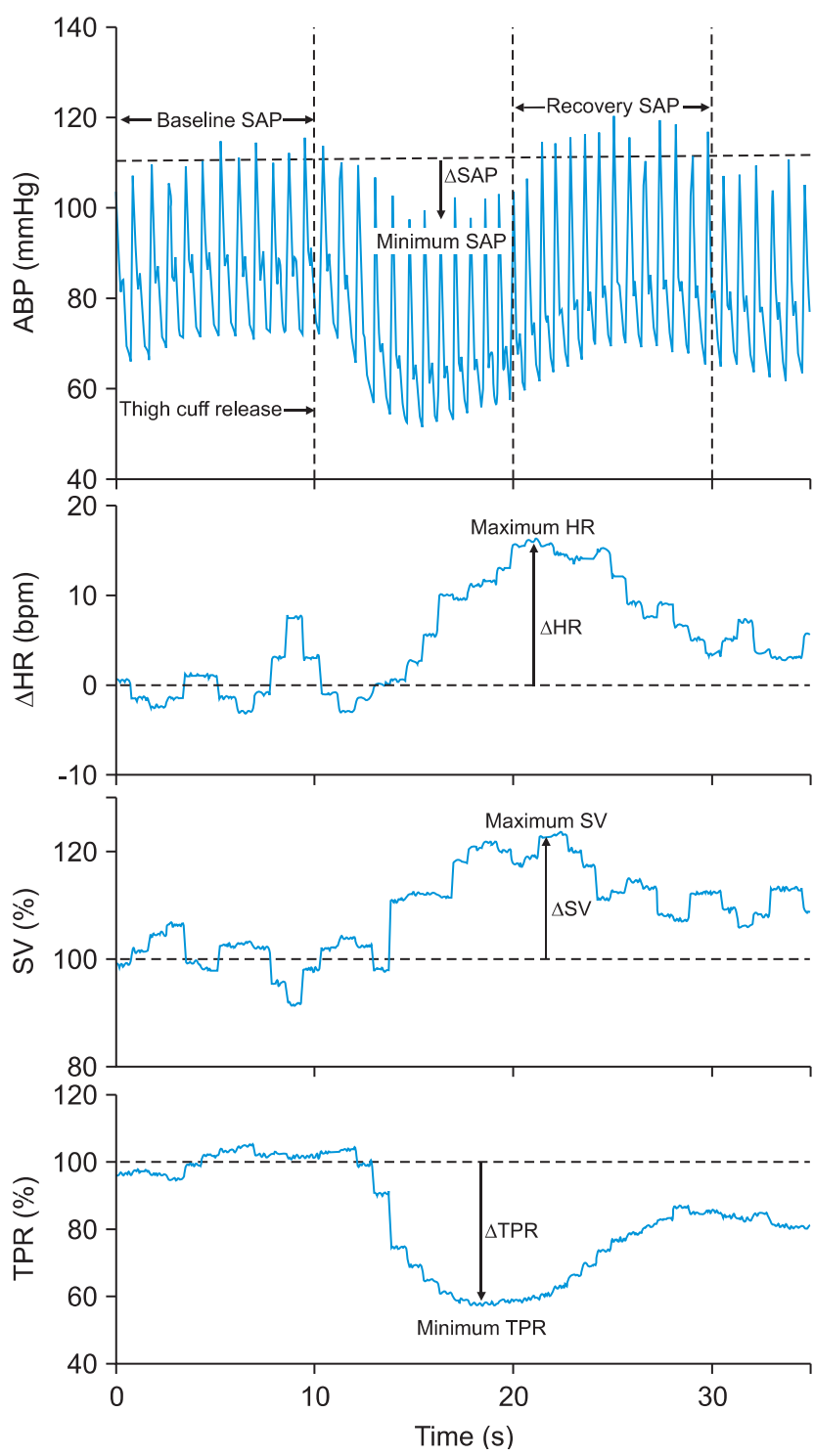

Fig. 2. Representative tracings of beat by beat changes in arterial blood pressure (ABP), heart rate (HR), stroke volume (SV) and total peripheral resistance (TPR) during thigh cuff release in one subject. Baseline values were obtained by averages during the $10 \mathrm{~s}$ before thigh cuff release. $\Delta \mathrm{SAP}, \Delta \mathrm{HR}, \Delta \mathrm{HR} / \Delta \mathrm{SAP}, \Delta \mathrm{SV}, \Delta \mathrm{TPR}$ and percentage restoration of SAP did not differ significantly between before and $60 \mathrm{~min}$ after melatonin ingestion. SAP: systolic arterial pressure. 
Changes in SV $(\Delta S V)$ and TPR $(\triangle T P R)$ were also calculated and expressed as percentages relative to their baseline values (Fig. 2).

\section{Dynamic CBF autoregulation}

Cerebrovascular resistance (CVR) was shown as CVR index
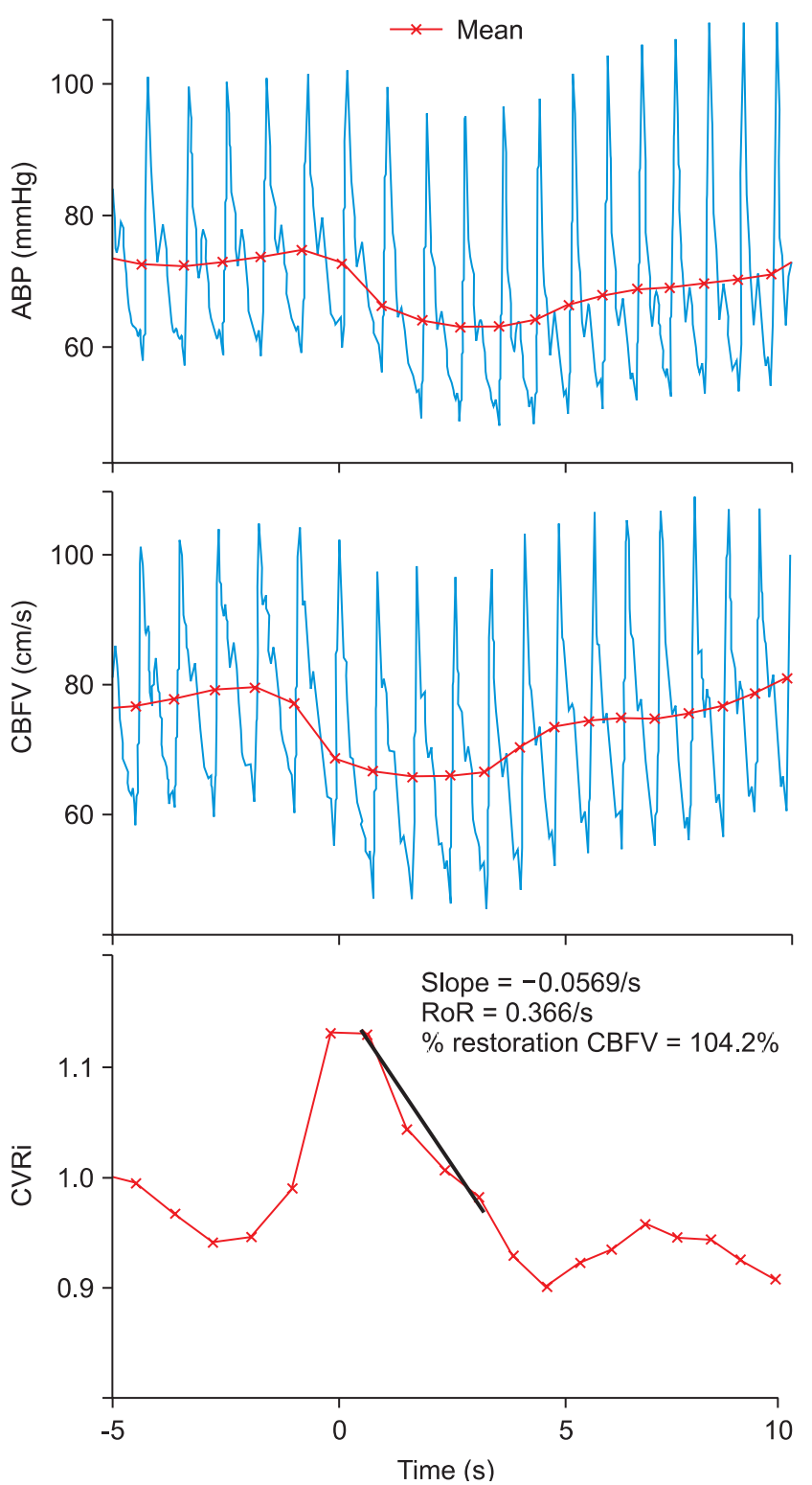

Fig. 3. Representative tracings of arterial blood pressure (ABP), cerebral blood flow velocity (CBFV) and beat-to-beat data of cerebrovascular resistance index (CVRi) during thigh cuff release in one subject. CVRi is shown in normalized units relative to control prerelease values obtained during -4 to $0 \mathrm{~s}$. The thigh cuffs were released at time 0 . The straight line through the CVRi curve (bottom graph) was determined by regression analysis of data in interval from 1 to $3.5 \mathrm{~s}$ after thigh cuff release and the slope of this regression line was used to calculate the rate of regulation. Melatonin did not affect CVRi or percentage restoration of CBFV before and $60 \mathrm{~min}$ after melatonin ingestion.
(CVRi) or MBP/CBFV. Control values for CVRi were determined by measuring the $4 \mathrm{~s}$ average instantly before thigh cuffs release. Changes in MAP, CBFV and CVRi during cuff release were estimated relative to their simultaneous control values. At 1.0 to $3.5 \mathrm{~s}$ after cuff release, the rate of change in CVRi is directly related to cerebral autoregulation without arterial baroreflex regulation [10]. Therefore, the rate of regulation (RoR) was calculated as an index of dCA from the slope of the regression line between CVRi and time $(T)$, normalized to cuff releaseinduced hypotension [10].

$\operatorname{RoR}=(\Delta \mathrm{CVRi} / \Delta T) / \Delta \mathrm{MAP} ;$

Where $\Delta \mathrm{CVRi} / \Delta T$ is the slope of the linear regression between CVRi and T; and $\triangle \mathrm{MAP}$, the gain of the step decrease, was determined by subtracting control MAP from averaged MAP during the interval from 1.0 to $3.5 \mathrm{~s}$ (Fig. 3). Normal RoR has been demonstrated to be around $20 \% \mathrm{~s}^{-1}$ (range, 15$30 \% \mathrm{~s}^{-1}$ ) [10]. Additionally, percentage restoration in CBFV was expressed as restoration/reduction $\times 100$ or [(recovery CBFV - minimum CBFV)/(baseline CBFV - minimum CBFV)] $\times$ 100 (\%), where baseline CBFV is the $4 \mathrm{~s}$ average value of $\mathrm{CBFV}$ before thigh cuff release; minimum $\mathrm{CBFV}$ is the nadir value of CBFV after thigh cuff release; and recovery $\mathrm{CBFV}$ is the average CBFV value between 6 and $10 \mathrm{~s}$ after thigh cuff release [16]. Therefore, we evaluated the two parts of the dynamic cerebrovascular responses: RoR reveals the early part ( $1-3.5 \mathrm{~s})$ of the vasodilatory response of dCA, and the later part (6-10 s) is reflected by percentage $\mathrm{CBFV}$ restoration.

\section{Statistics}

All pairwise comparisons of hemodynamic measurements before and after melatonin administration were assessed by paired t-tests or Wilcoxon signed rank test, as appropriate.

Using a pilot study and previous studies $[16,18]$, a power analysis revealed that a study size of at least 11 would be required to detect a $10 \mathrm{mmHg}$ and $15 \mathrm{~cm} / \mathrm{s}$ difference in means (SD 10) for the primary endpoint ABP and CBFV between control and melatonin with a power $80 \%$ at the $\mathrm{P}<0.05$ level of significance. A P value of $<0.05$ was considered statistically significant. All statistical analyses were performed using SigmaPlot 11.0 software (Systat Software, Inc., San Jose, CA, USA). Data are presented as mean \pm SD.

\section{Results}

Data on the thigh cuff method were analyzed in 11 of the 12 subjects, because one did not show $10 \mathrm{mmHg}$ decreases in $\mathrm{ABP}$ after thigh cuff releases. The mean \pm SD amount of melatonin administered were $13.1 \pm 0.9 \mathrm{mg}$. During the $60 \mathrm{~min}$ resting period after melatonin ingestion and before thigh cuff release, 
none of the hemodynamic variables, including HR, MAP, RR interval CBFV, CVRi, and end-tidal carbon dioxide, was altered significantly. Bispectral index, however, decreased 60 min after melatonin administration, although not significantly different $(\mathrm{P}$ $=0.057$ ) (Tables 1 and 2).

\section{Cardiovascular responses to thigh cuff release}

The average values of hemodynamic parameters and cardiovascular reflex indices are shown in Table 1 and Fig. 4. For the $60 \mathrm{~min}$ after ingestion of $0.2 \mathrm{mg} / \mathrm{kg}$ melatonin, SAP at rest did not change significantly $[124.8 \pm 10.5 \mathrm{mmHg}$ vs. $124.7 \pm$ $13.4 \mathrm{mmHg}, \mathrm{P}=0.989]$. Rapid release of the thigh cuffs elicited an abrupt decrease in ABP accompanying increases in HR and $S V$ and a reduction in TPR (Fig. 2). However, $\triangle \mathrm{SAP}, \Delta \mathrm{HR}, \Delta \mathrm{HR} /$ $\triangle \mathrm{SAP}, \Delta \mathrm{SV}, \Delta \mathrm{TPR}$ and percentage restoration of SAP did not differ significantly between before and $60 \mathrm{~min}$ after melatonin ingestion.

\section{Dynamic CBF autoregulation}

The average values of hemodynamic parameters and dynamic CBF autoregulation indices are presented in Table 2 and Fig. 4. For the $60 \mathrm{~min}$ following the administration of $0.2 \mathrm{mg} / \mathrm{kg}$ of melatonin, CBFV at rest did not change significantly $[58.4 \pm 11.7$ $\mathrm{cm} / \mathrm{s}$ versus $55.1 \pm 11.4 \mathrm{~cm} / \mathrm{s}, \mathrm{P}=0.182]$. Following the release

Table 1. Steady-state Hemodynamic and Respiratory Parameters and Dynamic Cardiovascular Responses to Thigh Cuff Release

\begin{tabular}{|c|c|c|c|}
\hline & Control & Melatonin & $P$ value \\
\hline \multicolumn{4}{|l|}{ Steady-state } \\
\hline SAP (mmHg) & $124.8 \pm 10.5$ & $124.7 \pm 13.4$ & 0.989 \\
\hline HR (beats/min) & $64.6 \pm 7.2$ & $64.9 \pm 8.0$ & 0.908 \\
\hline $\mathrm{SV}(\mathrm{ml})$ & $91.2 \pm 21.4$ & $86.6 \pm 12.1$ & 0.966 \\
\hline SV $(\%)$ & 100 & $98.7 \pm 19.4$ & 0.837 \\
\hline $\mathrm{CO}(\mathrm{L} / \mathrm{min})$ & $5.8 \pm 1.1$ & $5.6 \pm 0.9$ & 0.585 \\
\hline $\mathrm{CO}(\%)$ & 100 & $98.8 \pm 21.7$ & 0.861 \\
\hline TPR (dynes·sec/ $\mathrm{cm}^{5}$ ) & $1,363.7 \pm 268.0$ & $1,464.5 \pm 225.3$ & 0.392 \\
\hline TPR (\%) & 100 & $111.6 \pm 35.3$ & 0.638 \\
\hline \multicolumn{4}{|l|}{ Dynamic cardiovascular response } \\
\hline$\triangle \mathrm{SAP}(\mathrm{mmHg})$ & $14.6 \pm 4.3$ & $15.1 \pm 8.0$ & 0.845 \\
\hline$\Delta \mathrm{HR}$ (beats/min) & $17.9 \pm 4.4$ & $19.8 \pm 7.0$ & 0.395 \\
\hline$\Delta \mathrm{HR} / \Delta \mathrm{SAP}$ (beats $/ \mathrm{min} \cdot \mathrm{mmHg}$ ) & $1.2 \pm 0.4$ & $1.3 \pm 0.6$ & 0.449 \\
\hline$\Delta \mathrm{SV}(\mathrm{ml})$ & $15.5 \pm 10.1$ & $11.3 \pm 8.7$ & 0.156 \\
\hline$\Delta \mathrm{CO}(\mathrm{L} / \mathrm{min})$ & $3.2 \pm 2.5$ & $2.6 \pm 1.0$ & 0.409 \\
\hline$\Delta \mathrm{TPR}\left(\right.$ dynes$\left.\cdot \mathrm{sec} / \mathrm{cm}^{5}\right)$ & $600.6 \pm 232.8$ & $649.6 \pm 153.5$ & 0.523 \\
\hline Restoration of SAP (\%) & $117.9 \pm 31.6$ & $105.8 \pm 25.0$ & 0.233 \\
\hline \multicolumn{4}{|l|}{ BIS and respiratory parameters } \\
\hline BIS & $89.5 \pm 8.4$ & $83.5 \pm 8.3$ & 0.057 \\
\hline Pulse oxymetry (\%) & $98.1 \pm 1.0$ & $97.5 \pm 2.3$ & 0.300 \\
\hline End-tidal carbon dioxide ( $\mathrm{mmHg}$ ) & $38.5 \pm 3.3$ & $38.2 \pm 4.5$ & 0.825 \\
\hline
\end{tabular}

Values are mean \pm SD. SAP: systolic arterial pressure, HR: heart rate, $\triangle$ SAP: the reduction in SAP after thigh cuff release, CO: cardiac output, SV: stroke volume, TPR: total peripheral resistance. $\triangle$ HR: the response in HR after thigh cuff release, Restoration of SAP: percentage restoration of SAP in the interval from 20 to $30 \mathrm{~s}$ after thigh cuff release.

Table 2. Steady-state and Dynamic Hemodynamic and Cerebrovascular Responses to Thigh Cuff Release

\begin{tabular}{lccc}
\hline & Control & Melatonin & P value \\
\hline Steady state & & & 0.443 \\
MAP (mmHg) & $81.7 \pm 8.6$ & $54.6 \pm 8.5$ & 0.182 \\
CBFV (cm/s) & $58.4 \pm 11.7$ & $0.0437 \pm 0.02$ & 0.358 \\
Dynamic responses & & $0.187 \pm 0.07$ & 0.343 \\
Slope (/s) & $0.0380 \pm 0.01$ & $-18.1 \pm 5.2$ & 0.651 \\
RoR (/s) & $0.163 \pm 0.06$ & $-9.8 \pm 2.5$ & 0.201 \\
MAP response to cuff release (mmHg) & $-17.5 \pm 4.3$ & 0.681 \\
CBFV response to cuff release $(\mathrm{cm} / \mathrm{s})$ & $-8.6 \pm 2.0$ & $18.8 \pm 7.2$ & 0.162 \\
CVRi response to cuff release $(\mathrm{mmHg} / \mathrm{cm} / \mathrm{s})$ & $19.4 \pm 4.9$ & $77.2 \pm 27.8$ & \\
Restoration of CBFV $(\%)$ & $92.6 \pm 24.9$ & & \\
\hline
\end{tabular}

Values are mean \pm SD. MAP: mean arterial pressure, CBFV: cerebral blood flow velocity, CVRi: cerebrovascular resistance index, RestorationCBFV: percentage restoration in cerebral blood flow velocity in the interval from 6 to $10 \mathrm{~s}$ after thigh cuff release. 

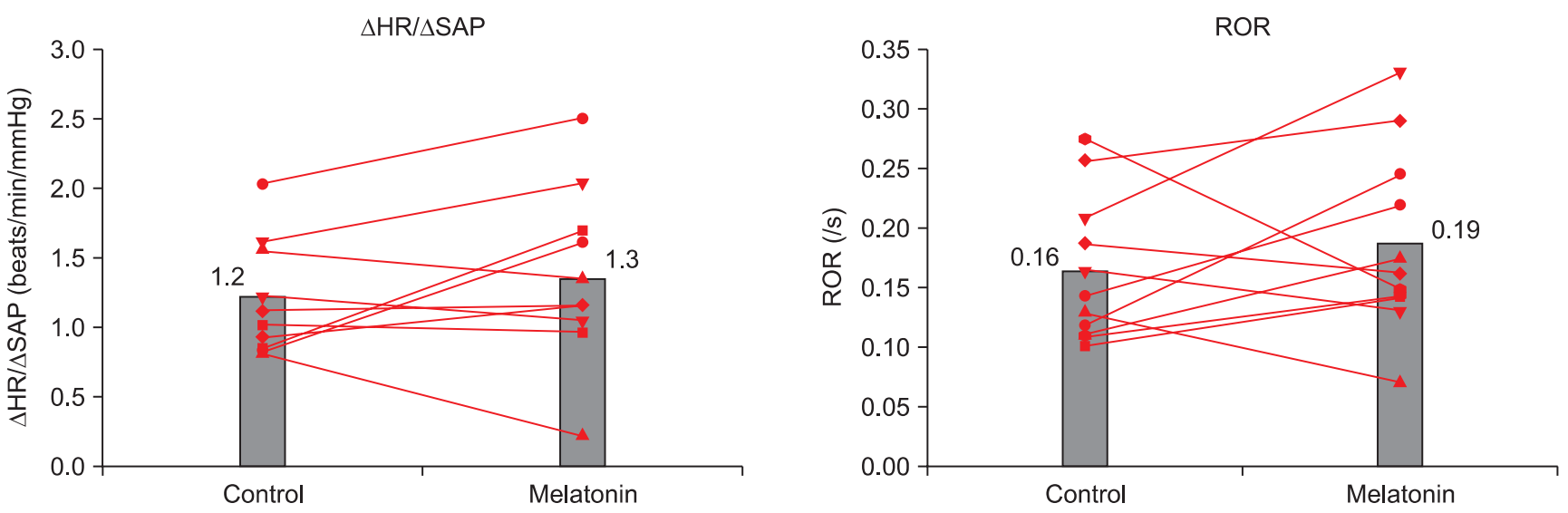

Fig. 4. Comparison of the index of arterial cardiac reflex $(\triangle \mathrm{HR} / \triangle \mathrm{SAP}$, left) and the index of dynamic cerebral autoregulation, rate of regulation (RoR, right) for each subject (plots) and median (bars) of each parameter before and after melatonin administration. Melatonin did not affect index of arterial cardiac reflex and RoR before and 60 min after melatonin ingestion. $\triangle$ HR: the response in HR after thigh cuff release, $\triangle$ SAP: the reduction in SAP after thigh cuff release.

of the thigh cuffs, the change in MAP was $-17.5 \pm 4.3 \mathrm{mmHg}$ before and $-18.1 \pm 5.2 \mathrm{mmHg}$ after melatonin administration $(\mathrm{P}=0.651)$. These decreases in ABP were sufficient to evoke transient decreases in CBFV and marked cerebral autoregulatory responses [10]. Reduction in CBFV did not differ significantly $[-8.6 \pm 2.0 \mathrm{~cm} / \mathrm{s}$ versus $-9.8 \pm 2.5 \mathrm{~cm} / \mathrm{s}, \mathrm{P}=0.201]$. Although the initial response to decreased $\mathrm{ABP}$ was a transient decrease in $\mathrm{CBFV}$, the cerebral autoregulatory response rapidly decreased the CVRi, thus preventing further decreases in CBFV (Fig. 3). Therefore, the return of CBFV to baseline was much faster than that of ABP. Melatonin did not affect CVRi, RoR or percentage restoration of $\mathrm{CBFV}$ before and $60 \mathrm{~min}$ after melatonin ingestion.

\section{Discussion}

Contrary to our hypothesis, we found that, when transient decrease in ABP was induced by rapid thigh cuff deflation, the reduction in SAP did not differ significantly before and 60 min after melatonin ingestion. In addition, changes in $\mathrm{HR}, \Delta \mathrm{HR} / \triangle \mathrm{SAP}, \mathrm{TPR}, \mathrm{SV}$ and the percentage restoration of SAP were unaffected by melatonin, indicating that melatonin premedication does not impair ABP preserving capability in response to sudden $\mathrm{ABP}$ perturbation. Additionally, in the cerebral circulation, melatonin did not affect changes in CBFV, CVRi, RoR and percentage restoration of CBFV following a sudden decrease in ABP, which suggeststhat melatonin does not affect rapid vasodilatory and recovery responses of the dCA.

We used a $0.2 \mathrm{mg} / \mathrm{kg}$ premedication dose of melatonin, which has been shown to reduce the doses of propofol and thiopental required for loss of responses to verbal commands and eyelash stimulation [2]. In contrast to previous findings, in which $1 \mathrm{mg}$ of melatonin decreased MAP in men and women $[5,6]$, we found that after ingestion of $0.2 \mathrm{mg} / \mathrm{kg}$ melatonin, $\mathrm{ABP}$ and $\mathrm{HR}$ at rest did not change significantly over the subsequent $60 \mathrm{~min}$. In agreement with our findings, however, 3 mg melatonin was shown to have no effect on $A B P$ at rest $[7,20]$, suggesting that the effects of exogenous melatonin on ABP in humans may be dose related $[7,8,20]$. Studies in animals also reported that vascular changes [21], adrenal nerve activity [22], and hormonal secretion responses [23] showed dose response relationship to melatonin concentration.

We observed no significant differences in SAP and HR responses during acute hypotensive stimuli before and 60 min after melatonin ingestion. In particular, changes in TPR during thigh cuff deflation were not affected, suggesting that vasoconstrictive responses were preserved after melatonin ingestion. This finding is consistent with previous results, which showed that, although melatonin attenuated muscle sympathetic nerve activity responses during orthostatic challenge induced by lower body negative pressure (LBNP), ABP, HR and forearm vascular resistance during LBNP were similar in the melatonin and placebo trials [8]. The lack of a reduction in ABP and increase after thigh cuff release could be the result of the melatonin-induced augmentation of noradrenalininduced vasoconstriction. Therefore, our findings, along with previous results, suggest that melatonin does not influence ABP preservation during sudden hypotensive stimuli induced by rapid thigh cuff deflation or during persistent hypotensive stimuli evoked by LBNP, although melatonin doses are different.

The thigh cuff technique to study the dynamic behavior of cerebral blood pressure autoregulation was introduced by Aaslid et al. [10]. When large pneumatic cuffs placed around both thighs are inflated above systolic pressure for $\geq$ 
2 minutes and then suddenly released, a sharp drop in ABP is usually observed, lasting $\sim 10$ seconds before returning to its original level. Rapid thigh-cuff deflation after three minutes of suprasystolic inflation is associated with a massive inflow of blood into the low resistance lower limbs, immediately lowering central arterial pressure. This accompanied by pooling of blood in the lower limb venous system reduces the veour return to the heart, further reducing central arterial pressure. Thigh cuff deflation typically reduces central artery blood pressure by 15$20 \%$ and restoration of normal pressure is not usually complete for $20 \mathrm{~s}$.

There is now convincing evidence that melatonin acts through two receptors, MT1 and MT2, in the vasculature. Activation of MT1 and MT2 receptors has been shown to elicit vasoconstriction and vasodilation, respectively [21]. Melatonin has been shown to vasoconstrict the tail and cerebral arteries in rats $[21,24]$ and to vasodilate aorta, iliac, and renal arteries [25-27]. Similarly, melatonin administration differentially altered vascular blood flow in humans, demonstrating that renal blood flow velocity and conductance were lower during the melatonin than during the placebo trial [20]. On the contrary, forearm blood flow and conductance were greater with melatonin than with placebo [20]. These differing vascular effects of melatonin are likely due to the relative distribution of MT1 and MT2 receptors, suggesting that melatonin has complex effects on human vasculature [20].

In rats, melatonin was shown to reduce regional CBF [24], and to directly constrict cerebral arteries through alteration of potassium channels [28]. In humans, however, ingestion of 3 mg melatonin did not result in a change in CBFV, as measured by transcranial Doppler [20], a finding in accordance with our results. Moreover, intravenous melatonin injection did not alter basilar artery blood flow, as determined by magnetic resonance imaging [11], providing further evidence that, unlike in rats, melatonin does not alter $\mathrm{CBF}$ at rest in humans.

Despite the changes in cerebral perfusion pressure between 60 and $150 \mathrm{mmHg}$, static cerebral autoregulation maintains CBF at relatively constant levels. However, CBF responds rapidly to temporal alterations in ABP even during normal conditions $[10,19,29]$. The regulatory capability of these rapid changes in CBF is defined as dCA. The thigh cuff method has typically been utilized to assess how rapidly cerebral vessels dilate and $\mathrm{CBFV}$ returns to baseline when $\mathrm{ABP}$ remains reduced during a short period of time. The RoR, measured by the normalized change in CVR per second during the $2.5 \mathrm{~s}$ period instantly after a transient reduction in $\mathrm{ABP}$, describes the rapid vasodilatory response of cerebral vasculature and the early part ( 1 to 3.5 seconds) of restoration in CBFV, i.e., prior to the possibility of an arterial baroreflex-mediated restoration of ABP [10]. We found that melatonin did not significantly alter RoR, suggesting that the early portion of the vasodilatory response in dCA had been preserved.

The later portion of restoration of CBF velocity was evaluated as the percentage restoration of CBFV. Our assumption, that restoration of CBFV ought to be nearly complete within 6-10 seconds following thigh cuff deflation, was based on the findings showing that CBFV was almost restored within this time during normal autoregulation $[10,16]$. We observed no significant difference in this index before and after melatonin ingestion, which indicates that melatonin does not compromise the restoration of $\mathrm{CBF}$ in response to rapid $\mathrm{ABP}$ perturbation.

This study had several limitations. First, although transcranial Doppler is widely used to detect changes in CBF, it measures CBFV of the cerebral artery rather than CBF. Blood velocity reflects blood flow only when the diameter of the blood vessel remains constant. In this regard, the diameter of the middle cerebral artery has been reported to show little change in response to acute hemodynamic perturbations [30], such as those elicited during the thigh cuff deflation protocol [10]. Second, we did not measure plasma melatonin concentrations, although ingestion of $3 \mathrm{mg}$ melatonin was shown to result in plasma melatonin concentrations more than 100-fold higher than endogenous daytime plasma melatonin concentrations [8]. Third, we did not measure muscle sympathetic nerve activity as a direct estimate of sympathetic response during hypotensive stimuli. Rather, continuous change in TPR, using the Modelflow method, was used as an index of vasoconstrictive response.

In conclusion, we have evaluated the effects of melatonin on dynamic cardiovascular and cerebral autoregulation assessed by the thigh cuff method. Our results suggest that melatonin does not impair ABP preserving capability and dCA in response to sudden hypotensive stimuli. These findings indicate that melatonin premedication may be safe under clinical conditions, such as postural changes, hemorrhage, and other operative stimuli, in which arterial pressure decreases temporally.

\section{References}

1. Wilhelmsen M, Amirian I, Reiter RJ, Rosenberg J, Gogenur I. Analgesic effects of melatonin: a review of current evidence from experimental and clinical studies. J Pineal Res 2011; 51: 270-7.

2. Naguib M, Samarkandi AH, Moniem MA, Mansour Eel-D, Alshaer AA, Al-Ayyaf HA, et al. The effects of melatonin premedication on propofol and thiopental induction dose-response curves: a prospective, randomized, double-blind study. Anesth Analg 2006; 103: 1448-52.

3. Chuang JI, Chen SS, Lin MT. Melatonin decreases brain serotonin release, arterial pressure and heart rate in rats. Pharmacology 1993; 47: 91-7.

4. Kawashima K, Miwa Y, Fujimoto K, Oohata H, Nishino H, Koike H. Antihypertensive action of melatonin in the spontaneously hyper- 
tensive rat. Clin Exp Hypertens A 1987; 9: 1121-31.

5. Arangino S, Cagnacci A, Angiolucci M, Vacca AM, Longu G, Volpe A, et al. Effects of melatonin on vascular reactivity, catecholamine levels, and blood pressure in healthy men. Am J Cardiol 1999; 83: 1417-9.

6. Cagnacci A, Arangino S, Angiolucci M, Maschio E, Melis GB. Influences of melatonin administration on the circulation of women. Am J Physiol 1998; 274: R335-8.

7. Cook JS, Ray CA. Melatonin attenuates the vestibulosympathetic but not vestibulocollic reflexes in humans: selective impairment of the utricles. J Appl Physiol 2010; 109: 1697-701.

8. Ray CA. Melatonin attenuates the sympathetic nerve responses to orthostatic stress in humans. J Physiol 2003; 551: 1043-8.

9. Tiecks FP, Lam AM, Aaslid R, Newell DW. Comparison of static and dynamic cerebral autoregulation measurements. Stroke 1995; 26: 1014-9.

10. Aaslid R, Lindegaard KF, Sorteberg W, Nornes H. Cerebral autoregulation dynamics in humans. Stroke 1989; 20: 45-52.

11. van der Helm-van Mil AH, van Someren EJ, van den Boom R, van Buchem MA, de Craen AJ, Blauw GJ. No influence of melatonin on cerebral blood flow in humans. J Clin Endocrinol Metab 2003; 88: 5989-94.

12. Huh IY, Park SE, Yang HS, Hwang GS. Modelflow method versus continuous thermodilution technique for cardiac output measurement in liver transplant patients. Korean J Anesthesiol 2008; 55: 5765.

13. Bogert LW, van Lieshout JJ. Non-invasive pulsatile arterial pressure and stroke volume changes from the human finger. Exp Physiol 2005; 90: 437-46.

14. Sugawara J, Tanabe T, Miyachi M, Yamamoto K, Takahashi K, Iemitsu $\mathrm{M}$, et al. Non-invasive assessment of cardiac output during exercise in healthy young humans: comparison between Modelflow method and Doppler echocardiography method. Acta Physiol Scand 2003; 179: 361-6.

15. Remmen JJ, Aengevaeren WR, Verheugt FW, van ver Werf T, Luijten HE, Bos A, et al. Finapres arterial pulse wave analysis with Modelflow is not a reliable non-invasive method for assessment of cardiac output. Clin Sci (Lond) 2002; 103: 143-9.

16. Ogawa Y, Iwasaki K, Aoki K, Kojima W, Kato J, Ogawa S. Dexmedetomidine weakens dynamic cerebral autoregulation as assessed by transfer function analysis and the thigh cuff method. Anesthesiology 2008; 109: 642-50.

17. Samarkandi A, Naguib M, Riad W, Thalaj A, Alotibi W, Aldammas F, et al. Melatonin vs. midazolam premedication in children: a double-blind, placebo-controlled study. Eur J Anaesthesiol 2005; 22: 189-96.

18. Kato J, Ogawa Y, Kojima W, Aoki K, Ogawa S, Iwasaki K. Cardiovascular reflex responses to temporal reduction in arterial pressure during dexmedetomidine infusion: a double-blind, randomized, and placebo-controlled study. Br J Anaesth 2009; 103: 561-5.

19. Zhang R, Behbehani K, Crandall CG, Zuckerman JH, Levine BD. Dynamic regulation of heart rate during acute hypotension: new insight into baroreflex function. Am J Physiol Heart Circ Physiol 2001; 280: H407-19.

20. Cook JS, Sauder CL, Ray CA. Melatonin differentially affects vascular blood flow in humans. Am J Physiol Heart Circ Physiol 2011; 300: H670-4.

21. Doolen S, Krause DN, Dubocovich ML, Duckles SP. Melatonin mediates two distinct responses in vascular smooth muscle. Eur J Pharmacol 1998; 345: 67-9.

22. Niijima A, Chun SJ, Shima T, Bizot-Espiard JG, Guardiola-Lemaitre B, Nagai K. Effect of intravenous administration of melatonin on the efferent activity of the adrenal nerve. J Auton Nerv Syst 1998; 71: 134-8.

23. Forsling ML, Wheeler MJ, Williams AJ. The effect of melatonin administration on pituitary hormone secretion in man. Clin Endocrinol (Oxf) 1999; 51: 637-42.

24. Capsoni S, Stankov BM, Fraschini F. Reduction of regional cerebral blood flow by melatonin in young rats. Neuroreport 1995; 6: 1346-8.

25. Satake N, Oe H, Sawada T, Shibata S. The mode of vasorelaxing action of melatonin in rabbit aorta. Gen Pharmacol 1991; 22: 21921.

26. Satake N, Oe H, Shibata S. Vasorelaxing action of melatonin in rat isolated aorta; possible endothelium dependent relaxation. Gen Pharmacol 1991; 22: 1127-33.

27. Satake N, Shibata S, Takagi T. The inhibitory action of melatonin on the contractile response to 5-hydroxytryptamine in various isolated vascular smooth muscles. Gen Pharmacol 1986; 17: 553-8.

28. Geary GG, Krause DN, Duckles SP. Melatonin directly constricts rat cerebral arteries through modulation of potassium channels. Am J Physiol 1997; 273: H1530-6.

29. Aaslid R, Newell DW, Stooss R, Sorteberg W, Lindegaard KF. Assessment of cerebral autoregulation dynamics from simultaneous arterial and venous transcranial Doppler recordings in humans. Stroke 1991; 22: 1148-54.

30. Giller CA, Hatab MR, Giller AM. Estimation of vessel flow and diameter during cerebral vasospasm using transcranial Doppler indices. Neurosurgery 1998; 42: 1076-81. 